normal. As there was no return of fever, and he continued to feel quite well, he returned to school on the 14th. Subsequently it was ascertained that he felt slight rheumatic pains in the arms during the remainder of the week. On the 17th the boy complained of headache and of pain in the back of the neck. The pain radiated up from the first dorsal spine over the occiput; the headache was frontal. The temperature was $101 \cdot 6^{\circ}$, and there were vomiting and constipation, with a very coated tongue. Later in the day there were pain and weakness in the left arm, which soon after midnight was completely paralysed.

When the patient was seen on the 18th there was complete loss of power in the left shoulder and upper arm, and the forearm and hand preserved only a very slight power of flexing and extending the fingers. There was no change in this condition throughout the progress of the case. There was no alteration of sensation in the limb and the reflexes were absent. The left naso-labial fold was not so well marked as the right, and subsequently slight muco-purulent discharge developed in the left eye, but I was never able to say definitely that there was left facial paralysis, nor could the boy's relatives detect any change in his a ppearance. A faint extensor plantar reflex was obtained on the left side, but no weakness of the legs could be detected at any time, though later in the case there were twitching and constant restless movement of both lower extremities. There was some difficulty in micturition, but this passed off and no incontinence or retention was observed throughout. 'The deep and superficial reflexes below the level of the dorsal enlargement were normal. Photophobia was a prominent symptom, but there was no optic neuritis, nor did it develop subsequently. The vomiting, headache, and constipation persisted, but the pain over the upper part of the spine, which had not been relieved by aspirin, phenacetin, or counter irritation, was apparently stopped by the application of an ice-bag. Any attempt to flex the head forward on to the chest awakened the pain afresh. The temperature during this and the subsequent day varied between $103^{\circ}$ and $104^{\circ}$, the pulse was 120 , and the respirations were 30 .

During the night of the 18th the shoulder and scapular muscles of the right arm became paralysed; the upper arm, forearm, and hand muscles were intact. During the two following days the fever kept high, and at intervals there was delirium. The photophobia and pain in the neck were less marked, but there was a pain in the lumbar region extending round the abdomen. There were also epistaxis and constant restless movement of the legs. The sphincters were not affected. On the 21st the temperature fell to normal and remained so. The respiration became for a short time slower, but was altered in character, being purely costal in type, whilst the reversed movement of the abdominal wall pointed to paralysis of the diaphragm. From this point the mental condition became worse, fæces, and later on urine, were passed into the bed, and the twitching and restlessness of the lower limbs were more observable. The patient died on the sixth day of the illness from gradual respiratory failure. On the day of his death there was no paralysis of the lower extremities, no loss of sensation, or alteration of deep or superficial reflexes, except a doubtful extensor reflex on the left side. The pupils were equal in size and contracted; there was partial control over the bladder.

The points of especial interest are : the existence of a brier, sharp febrile attack one week before the onset of the illness; this fact, coupled with the nature of the pyrexia and with the occurrence of epistaxis, would point to the disease being of infective origin. The existence of slight facial paresis has been noted before in cases of infantile paralysis. It is generally transitory, and indicates that the condition is not a purely local spinal affection, but that other parts of the nervous system may be affected. There is a steadily accumulating weight of evidence to show that the direct exciting cause of acute anterior poliomyelitis attacks the whole nerrous system with a special predilection for the grey matter (owing perhaps, as Buzzard suggests, to its greater vascularity). The resulting symptoms will, of course, vary according to the site of the lesion; sometimes they are cerebral, sometimes spinal, and sometimes neuritic. In the cerebral group there may be further variations according to the relative severity of the lesions in the cortex or in the basal ganglia. It is clear, therefore, that to regard the disease as spinal in its distribution is to take too limited a view; it should rather be thought of as a general infection of the nervous system.

Clifton, Bristol.

\section{CHRONIC SPHENOIDAL SINUS DISEASE.}

BY W. S. SYME, M.D. EDIN.,

ASSISTANT SURGEON, EAR, NOSE, AND THROAT HOSPITAL, GLASGOW.

THIs communication is based on a total of 17 patients on whom I have operated for disease of one or of both sphenoidal sinuses during the past 18 months. In most of them the posterior ethmoidal cells were also involved, but in the number are not included cases in which the sphenoidal condition was associated with antral or frontal sinus disease, nor those where the general nasal condition was one of atrophic rhinitis with ozæna. In most of these patients, as well as in others who did not submit to operation, an ophthalmological examination was obtained, and in this connexion my thanks are due to Dr. John Rowan, the consulting oculist to the Glasgow Ear, Nose, and Throat Hospital. In all the disease was of the chronic variety and in nine it was limited to one side. Though several acute cases have come under my care in none of them have $I$ found it necessary to operate, cure being obtained by nasal douching with or without lavage of the sinus itself. I propose first shortly to relate the chief facts in connexion with these cases, and then, with them as a text, to make some observations on sphenoidal sinus disease.

CASE 1.-A male, aged 50 years. Nasal obstruction and nasal discharge for some months. Disease of left sphenoidal sinus and of left posterior ethmoidal cells. Ethmoidal cell above and external to the sinus. Eyes-Right : disc pinkish and full; marked venous pulsation. Left: disc pale, suggesting optic atrophy, which is borne out by further examination. Result: Nasal condition cured; sinus quite dry; patient feels quite well. Eye condition progressive on the left side. CASE 2.-A male, aged 39 years. Discharge from nose of one year's duration, sore throat, hoarseness at times, deafness and noises in right ear of two weeks' duration, severe giddiness for same length of time. Ears-Right: membrane shows cicatrices from former middle-ear suppuration; quite dry; wateh 0 , Rinné ${ }^{\text {. }}$ Left: mernbrane shows adhesive processes and fibrous changes in tympanum; watch 6 , Rinné + . Vertigo severe, with tendency to fall to right side. Quite unable to walk without assistance. Nystagmus on looking to either side, worse on looking to right, horizontal in direction and strongest movement towards side to which eyes are turned. No nystagmus when looking straight forward. Caloric reactions: Cold wate in the right ear does not appreciably alter nystagmus in either direc tion. Warm water in right slightly increases nystagmus to right; does not appreciably change nystagmus to left. Cold water in left ear.
abolishes nystagmus to left and much increases nystagmus to right. Warm water in left ear abolishes nystagmus to right and much Warm water in left ear abolishes nystagmus to right and much
increases nystagmus to left. Patellar reflexes increased, no ankle increases nystagmus to left. Patellar reflexes increased, no ankle
clonus ; right plantar reflex increased but still flexor. Nose: Right clonus ; right plantar reflex increased but still flexor. Nose : Right polypi, granulations, and pus in outer and upper part. At the anterior polypi, granulations, and pus in outer and upper part. At the anterior
part of the supero-external boundary the granulations could be seen to be pulsating. A probe gently pressed against this part allowed some be pulsating. A probe gently pressed against this part allowed some
drops of pus to enter the sinus. Eyes : Neuritis in both. Course: This drops of pus to enter the sinus. Eyes : Neuritis in both. Course: This patient improved slowly; the sphenoidal cavity required a good deal of tions. The vertigo immediately began to improve after the operation on the sinus, but he had occasional severe attacks which appeared to be dependent on blocking of the sinus outlet. Now the discharge bas quite ceased. Once or twice when he has sniffed strongly he has had a slight vertiginous attack, but otherwise he has had no decided vertigo for several months. A feeling of dizziness remains, especially if he stoops or turns suddenly. He looks well and expresses himself as feeling so, and is fit for work. The hearing in the right ear has improved without other treatment, the watch is now heard at three inches, the higher tuning forks are heard rather better than the lower, and he says that the note of the tuning fork is discordant when compared with the left ear. There is still nystagmus, chiefly when the eyes are directed to
the right, very slight when turned to the left. The optic neuritis is passing off, the improvement being more marked on the left side.

CASE 3.-A female, aged 45 years. Post-nasal discharge of two years duration with severe occipital headaches and feeling of general illhealth. Double sphenoidal sinusitis and posterior ethmoidal disease. Lining membrane oedematous along outer wall of right sinus, which is small. Odematous membrane with polypi and granulations in extends above and to the outer side of sphenoid for a considerable distance. Granulations in this cell and in adjacent parts of sphenoid. Eyes: Neuritis in both, more marked in left. in general health; neuritis in eyes passine;

CASE 4.-A male, aged 36 years. Post-nasal discharge of long duration with sore throat. Sphenoidal and posterior ethmoidal disease on both sides. (Edematous and polypoidal lining membrane in both Eyes: Discs full, edges indistinet, neuritis. Result: Disappeared from observation.

CASE 5.-A female, aged 35 years. Discharge from nose of one year's duration, severe headaches. Pus and polypoidal tissue in right sphenoidal sinus, polypoidal tissue in antero-external recess of left side. Eyes : Dises pinkish and full; veins full and twisted, the left side being
the worse. Result : Nasal condition cured, no discharge, general health 
much better, headaches less frequent and not so severe. Eyes : Condition passing off.

CASE 6. - A female, aged 23 years. Discharge from nose of one year's duration, general ill-health. Double sphenoidal and posterior ethmoidal disease. Ethmoidal cells on both sides full of polypoidal and granula tion tissue. Pus in right sinus which is small. Left sinus larger and contains polypoidal tissue in the part adjacent to ethmoid. Eyes: Discs
pinkish; veins, especially in right, full and twisted. Result: Cured; pinkish; veins, especially in right, full and twisted. Result: nose free from discharge ; patient feels quite well ; eyes normal.
CASE 7.-A male, aged 45 years. Much post-nasal discharge of four or five years' duration, headaches for some time chiefly on right side.
Right sphenoidal disease, sinus small and full of polypoidal tissue. Fyes not examined. This case was operated on without removal of the middle turbinate. Result: Cured; discharge quite ceased and headaches gone.

CASE 8.-A male, aged 47 years. Nasal obstruction of four years' duration, loss of sense of smell, nasal polypi in both nostrils. Double ethmoidal and sphenoidal disease. Polypi in left sphenoidal sinus, Result: Disappeared from observation.

CASE 9.-A female, aged 22 years. Post-nasal discharge of two years' duration, general ill-health. Right sphenoidal and posterior ethmoidal disease. Pus and granulations in right sphenoidal sinus. Posterior ethmoidal cell external to sinus in practically its whole length. Eyes normal. Result : The nose in this case is very narrow and the aftertreatment was protracted. Now the patient feels much better in health, but the discharge, though much less, has not altogether ceased. CASE 10.-A female, aged 47 years. Nasal obstruction and discharge of two years' duration. Left sphenoidal and posterior ethmoidal disease. Large development of ethmoidal cells and of sinus. Posterior wall of sinus $4 \frac{1}{4}$ inches from nasal spine. Ethmoid much diseased, granulations and polypoidal tissue in sinus adjacent to ethmoid. Fyes mormal. Result : Recent case; cure of nose proceeding satisfactorily.

charge, and sore throat for two or three years. Double sphenoidal disdisease. Right side operated on nine months a abo. Opheration sinus disease. Right side operated on nine months ago. Operation on left recently. Edematous lining membrane in right sinus, good-sized polypus at entrance to left sinus, and polypoidal condition of lining membrane chief y in foor. Fyes: Dises pink and full, more marked on
right. Result : Right side cured, free from discharge; left side proceeding satisfactorily.

CASE 12. - A female, aged 23 years. Nasal obstruction on right side of six years' duration, frequent headaches. Polypi in right nostril. Disease of right sphenoidal sinus and right posterior ethmoidal cells.
Eyes normal. Result: Nasal condition cured, headaches have dis. Eyes normal. Result: Nasal condition
appeared, and the patient feels quite well.

appeared, and the patient feels quite well.
C $A S E$ 13.-A male, aged 46 years. Post-nasal discharge of some years' duration. During the past two years the discharge has changed in character, having become more purulent and taken on a sickly odour. Feeling of malaise and depression of spirits, sore throat, hoarseness at times, dull headache with occasional acute headache over left frontal cegion. Treated for several years without benefit by various means for post-nasal catarrh. Left sphenoidal and ethmoidal disease. Soft polypoidal tissue and bright yellow pus in ethmoid. Large posterior ethmoidal cell full of diseased tissue extending somewhat above sphenoidal sinus. This latter cavity was of large size and full of except at the part next to the ethmoidal cell, where there were a earious oxcept at the part granulations the lining membrane was quite carious opening and soft granulations, the lining membrane was quite healthy to the right side. Eyes normal. Result: Nasal condition cured, complete cessation of discharge, and disappearance of headaches and of plete cessation of discharge, and

CASE 14.-A female, aged 20 years. Post-nasal discharge with disagreeable odour of some vears duration; ill health; posterior spheagreeable odour of some years duration; ill health; posterior sphe-
noidal and ethmoidal disease on both sides. Small sinuses situated noidal and ethmoidal disease on both sides. Small sinuses situated treatment the condition is practically cured; the discharge and odour have almost entirely disappeared and the patient's health is quite have alm

CASE 15.-A female, aged 35 years. Frequent colds in head and postnasal discharge for some considerable time. Headache, especially on right side, and pain in right eye and right side of nose, with swelling, for the past week, of the tissues around the right eye; sore throat for some months. Right sphenoidal and ethmoidal disease. After removal of middle turbinate and curettage of ethmoid pus was seen coming from sphenoidal ostium. The sinus was opened and the lining was found to be diseased. Fyes: Discs normal, but there is sligh orbital cellulitis. Result: The pain in the head has ceased, the cellulitis has disappeared, and the nasal condition is on a fair way to cure. The case is a somewhat recent one.

CASE 16. - A female, aged 20 years. Discharge from nose, chiefly postnasal, of five years' duration; sickly odour at times. Headaches lately. Double sphenoidal and posterior ethmoidal disease, left side the worse. Operation on left side. Narrow sinus antero-posteriorly with formation of septa. Pus and odematous lining membrane chiefly in floor. Somewhat difficult case owing to irregular nasal septum. Eyes: Dises pinkish, veins full. The result of this case is yet undecided.

CASE 17. - A female, aged 26 years. Sore throat and post-nasal discharge of one year's duration. Hoarseness for some months. Left sphenoidal and posterior ethmoidal disease. Large posterior ethmoidal chiefly in sinus. (Edematous membrane and muco-pus far back on foor. Fyea normal. Recent case.

The chief complaint made by the patients was of continued post-nasal and nasal discharge and chronic head cold in some of several years' duration. Certain of them stated that the discharge had undergone a change in character, had become yellow and more thick, and had taken on a disagreeable odour. The results of this discharge were seen in more or less well-marked pharyngitis, granular, congestive, atrophic, or a combination of these ; laryngitis, and certain middle-ear changes which one associates with post-nasal discharge and its consequences. Headache was a common complaint; in three it was described as severe, in one the pain was confined to the side of the disease. Most of the patients also complained of depression of spirits, lack of energy, and a feeling of general seediness and ill-health.

The rhinological appearances were fairly constant. In one case only, a double sphenoidal sinusitis, were there ordinary nasal polypi present. Atrophic changes, not of a marked character and affecting chiefly the posterior parts of the inferior and middle turbinals, were observed in most. In several, however, the other extreme, cdema of these parts, was seen. In some the suggestion of disease in the posterior group of accessory air spaces was obtained by the presence of pus between the middle turbinate and the septum, but this sign was not present in all. In most of the cases after, if not before, the application of cocaine and adrenalin to the nasal mucosa a portion of the anterior wall of the sphenoidal sinus could be seen and examined, median rhinoscopy occasionally being helpful. This part was observed in some cases to be highly congested, and in others to be markedly anæmic although untouched by the anrsthetic, and in most some thin, sticky, purulent discharge could be wiped from its surface, and a probe passed into the crevice between the middle turbinate and the sinus dislodged some thicker pus. As a rule, however, the discharge was by no means large in amount. Posterior rhinoscopy, sometimes very difficult to carry out by reason of the increased irritability of the pharynx, showed intense congestion of the mucous membrane covering the vault, with purulent or muco-purulent discharge in this region and in the naso-pharynx generally. In some of the patients there was cedema of the septum posteriorly, but this condition is not uncommon apart from posterior nasal sinus disease.

None of the symptoms and signs which have been described are taken to be either separately or collectively pathognomonic of sphenoidal disease, but rather, one would say, are they suggestive of disease in the posterior group of nasal accessory cavities. To complete the diagnosis it is necessary to examine the inside of the sinus. To this end, after the application of cocaine and adrenalin to anæsthetise and contract the nasal mucous membrane, a probe, bent slightly at the point, is passed in an upward and backward direction between the middle turbinate and the septum, bisecting the length of the former. Arrived at the anterior wall of the sphenoid the point is turned upwards and outwards, and with a little patience and practice it is in most cases not difficult to pass it into the sinus. Sometimes, however, a good deal of difficulty is experienced and occasionally it is impossible, and this for anatomical reasons. In a series of 230 skulls examined I found the ostium absent in one large sinus, and in another, also of good size, it led into the posterior ethmoidal cell. It is necessary to bear in mind the average distance of the posterior wall of the sinus from the anterior nasal spine of the superior maxilla so as to decide if the probe has actually entered the cavity. I found this in adult skulls, male and female, to be $3 \frac{1}{2}$ inches, the extremes being $2 \frac{7}{8}$ and $4 \frac{1}{4}$ inches. In very few was the distance under 3 inches. These figures are in agreement with other observers. The passage of the probe should always be controlled by measurement or a graduated probe used. The posterior ethmoidal cell is a source of error in this connexion. In one patient $I$ found the posterior boundary of this cell on one side distant $3 \frac{1}{2}$ inches from the anterior nasal spine. It is very rarely, however, that it extends so far beyond the anterior wall of the sphenoid. As a rule, when the probe has passed into the sinus one has the sensation of its having entered a cavity and it can be moved about freely, though it must be borne in mind that the size of the sinus varies greatly. The condition of the lining membrane can now be ascertained to a certain extent. The probing should be done gently, especially in the upper and outer part, though it should be carried out systematically, for, as these cases show, the pathological changes may be strictly localised. The firm tap of the healthy parts can easily be distinguished from the sensation of bogginess which proceeds from odematous or polypoidal portions of the lining membrane. On the withdrawal of the probe and after wiping away all discharge from the neighbourhood a smallsized cannula may be passed, and with an air bag an attempt may be made to blow out any discharge there may be in the cavity, or syringing may serve the same purpose. As a rule, in chronic cases at any rate, only a small amount of discharge 
will be obtained. Its absence, however, has little import. ance; in several cases where there were found on operation well-marked changes in the lining membrane in the shape of granulation tissue or polypi, there was very little discharge actually present in the sinus. There is no doubt, I think, in chronic sphenoidal sinusitis, where as a rule the ostium is more patent from atrophic changes in its neighbourhood, that the discharge is constantly removed by syphonage and by thealterations in the position of the head, as for instance during lying and bending. This I take it is the reason why one has been so frequently told in these cases that the discharge is worse in the early part of the day. The same, as one knows, is the case in antral disease. The absence of pus on washing or blowing out the sphenoidal sinus has erroneously been taken as conclusive of the absence of disease. Some recommend puncture of the sinus by trocar and cannula through the anterior wall. This procedure is not free from risk, though the attachment of a stop to the cannula minimises this. The roof of the sinus sometimes dips very abruptly with an $\mathbf{S}$-shaped bend, and might conceivably be injured. In several skulls and in two patients the sinus of one side has extended well beyond the middle line anteriorly, and in such the puncture might be made into the wrong cavity. Personally, I have never found the necessity for its performance. Occasionally, when some difficulty has been experienced in passing the cannula through the ostium, the exertion of a little pressure when the point is in position has caused it to penetrate the mucous membrane, which partially occludes the natural opening, or the thin bone in the vicinity and to pass into the sinus. If the difficulty should be great it is well to wait till the removal of the middle turbinate has been carried out, if the examination already conducted warrants this operation. As a result of this proceeding the exploration of the sinus is much simplified, more of the anterior wall is seen, and any granulation or small polypi in the spheno-ethmoidal recess can be removed.

In one or two of the patients the examination of the anterior wall of the sphenoid with the probe gave the impression of bare bone, but no conclusion as to the inside of the sinus could be drawn from this, and, moreover, in certain parts of the nasal cavity where the membrane is thin it is easy to imagine that the bone is denuded of its covering.

Sondermann's suction apparatus is sometimes helpful in diagnosis. In one of my patients the removal of pus from the sphenoidal sinus was very evident. Its use, however, as a diagnostic, as well as a therapeutic, agent is not free from danger, in view of the possibility of the destruction of bone in the wall of the cavity in the region of the cavernous sinus.

In several cases Dr. W. F. Somerville kindly undertook the examination of the sphenoidal cavity and ethmoidal cells by skiagraphy, but I cannot say that we obtained any definite help from this method; certainly not so much as in some cases where $I$ had his help in which the question of frontal sinus disease was under consideration. Looking to the conditions found on operation this is not surprising.

In none of my cases were there urgent symptoms, such as those pointing to cavernous sinus thrombosis.

Even after the most careful examination by all methods and after weighing the symptoms it is in many cases impossible to arrive at a more definite diagnosis than that there is disease in one or more of the posterior ethmnidal cells or in the sphenoidal sinus, or in the whole of the posterior group of accessory air spaces. Even the discovery of changes in the optic nerve, bearing in mind the variation in the relation of the sinus to the posterior cell and of both to the nerve, will not help us much, though one's attitude towards operation will be modified thereby. The exact diagnosis must then in many cases remain in abeyance. As a matter of experience, however, I should say that though it is not unusual to get posterior ethmoidal disease without sphenoidal disease it is rare to get the latter without the former: or to put it in another way, chronic sphenoidal disease is, in my opinion. in most cases secondary to disease in the posterior ethmoidal cells. With acute sphenoidal disease this is probably not so commonly the case.

Ireatment. - As in disease of the antrum and frontal sinus so in disease of the region under consideration, certain cases either on account of their symptoms or becanse of the result of examination at once suggest the advisability of operation.
Probably, however, in the majority milder measures should first be given a trial. Of these, nasal douching is the chief followed by drying and insufflation of powdered boracic acid. In certain cases where the ostium is easily entered the sinus itself should be regularly washed out. The cavity is then dried by means of the air bag and boracic acid blown in through the cannula. Reference has already been made to the use of Sondermann's suction apparatus as a therapeutic method.

Besides the patients on whose cases this communication is based at least an equal number have come under my care in whom the diagnosis of sphenoidal disease was hardly doubtful and who have been treated without operation. When these simpler methods fail, or when for some reason their use is not considered advisable, there are several lines of surgical procedure open to us. In most cases removal of the middle turbinate is necessary if this has not already been carried out for purposes of diagnosis. The condition of the posterior ethmoidal cells can then be ascertained in more detail, and the removal of this structure is itself sometimes sufficient to admit of the cure of ethmoidal and sphenoidal disease by giving freer drainage and by permitting of the better application of simple antiseptic treatment. If, however, it is decided to deal with the ethmoidal disease by further operative measures, and if the state of the sphenoidal sinus should still remain in doubt, operative treatment should be limited to the cells. These can be removed by punch forceps and curette, acting, of course, under the control of good reflected light. That should be a matter of course in all intranasal surgery, but it is doubly imperative in dealing with these parts. It used to be recommended that curettage of the ethmoid should be carried out under the control of the finger passed behind the palate and into the posterior naris, but I hardly think any rhinological surgeon would consider this a sufficient safeguard. The curetting should be systematically and carefully performed, working always downwards and forwards. The opening up of the ethmoidal cells may reveal the pathway of the disease to the sphenoid. This is not unusually the case, and has been my experience in several of the patients operated on. In such one naturally follows the course which the pathological process has taken, opening into the sinus by way of the posterior ethmoidal cell. Where, however, the ethmoid has been dealt with without revealing a track to the spheroid it is well in such cases as we are now dealing with-viz., those in which the condition of the sinus remains in doubt-to await the result of this treatment on the discharge before proceeding further. The after-treatment should be carried out in the way to be later described.

When it is determined, $a b$ initio, to operate on the sphenoidal sinus this may in some few cases be performed without removal of the middle turbinate, the atrophy of the parts being sufficient to expose most of the anterior sphenoidal wall. As a rule, however, and especially in view of the frequency of associated ethmoidal disease, it is better to remove this structure as a preliminary. I then prefer to enter the sinus directly. This I do with a longbandled spoon passed to the region of the ostium, breaking through the posterior ethmoidal cell for the purpose if necessary. By a little pressure the bone in the neighbourhood is broken through, the opening is enlarged by working towards the centre and downwards, and the anterior wall is completely removed by means of Hajek's strong punch. I have used a long chisel to remove the lower thick part, but this punch seems strong enough for this purpose and is safer. For free drainage it is necessary that no cul-de-sac should be left in the lower part of the cavity. The extent of the sinus should now be determined by means of the probe and its walls carefully examined for diseased lining membrane and for carious openings in the bone. Special care and attention should be paid to the supero-external portion and to the recess adjacent to the ethmoid. In the former position the bone separating the cavity from the cavernous sinus, the carotid, and the optic nerve, is always thin, even if it is not absent through carious destruction or developmental defect, and in the latter $I$ have most frequently found œdematous and polypoidal lining membrane. The curettage should be carried out in detail, great gentleness being used in connexion with granulations growing in the neighbourhood of carious bone. Sometimes it is wiser to leave these untouched. To force should be employed in curetting the roof or external wall. Healthy portions of the lining membrane should be 
left, as by so doing the ultimate cure is hastened. If it is intended to remove the posterior ethmoidal cells this can now be done with safety, taking one's bearings from the external boundary of the sinus. In most cases the bone bounding the antero-external recess of the sinus is hard and firm, affording protection to the optic nerve as it passes through the foramen, but sometimes the cavity extends beneath this opening, and this prolongation may have a very thin roof or the roof may be in part absent. Needless to say, in such cases the curetting should be carried out with the greatest circumspection. In three skulls I found this anatomical peculiarity and in another skull the posterior ethmoidal cell extended similarly beneath the foramen.

Fortunately, I have never had the mischance to wound the cavernous sinus, though I am aware that this has happened to others. In the general run of cases the curettage of the cavity is not attended with severe hæmorrhage. There may be a sharp spurt for a minute or so, but this can be controlled by cotton-wool mops, and the operation can be carried out in detail and under the control of sight. If an opening is found in the intersinusal septum it will be necessary to do the double operation, even if this has not been determined upon by reason of the examination of the opposite nasal fossa, for the two cavities may be discharging through the one side. The parts may be swabbed with zinc chloride, silver nitrate, or some such solution. It is then carefully dried and boracic powder, or aristol and boracic powder, as is my habit, insufflated. No packing is required or desirable. The after treatment, both of the sphenoidal and of the ethmoidal region, exacts a good deal of patience and constant attention. Granulations grow quickly and tend to close the sinus before the interior is healed, so that they must be kept in check. Hence it is necessary at the operation to remove the anterior wall as completely as possible. In the ethmoidal part, too, granulations tend to be exuberant. Thoroughness in removing the disease from the sinus and cells will shorten the period of after-treatment, but it is always of some weeks' duration, and healing may not be complete for three or four months. There need, however, be no pessimism as to the result. The cases herein detailed prove this. The disagreeable post-nasal discharge ceases, the headaches are improved or disappear, and patients express themselves as feeling in every way more fit and cheerful. With many people the continuance of nasal or post-nasal discharge has a most depressing effect. They cannot keep their minds off it; it becomes almost their absorbing thought. A business man, for instance, stated that since the cure of his nasal disease he felt much more keen mentally. Previously the difficulty of concentrating his attention on any matter worried him. He was always conscious of the discharge and he felt depressed and melancholy. One has, of course, heard the same story in connexion with disease in other of the accessory nasal cavities, and, indeed, wherever a chronic nasal discharge is present.

Beyond some discolouration and puffiness of the skin in the region of the orbit and some rise of temperature in certain of the patients operation has been followed by no serious results. In none have I felt justified in using the external route. Occasionally it is necessary to remove a spur, to treat a septal deflection, or to do a partial tubinectomy as a preliminary. Even after doing this there is in some narrow noses such a confined space to work in that if there should be any severe symptoms or marked changes in the optic nerve it would, I think, be wise to operate as one does in the ethmoidal part of Killian's complete frontal operation. There is no doubt that this gives a very direct access to the ethmoidal cells and to the sphenoidal sinus, but sometimes the resulting scar is unsightly.

The considerations as regards anæsthesia are the same as in other intranasal operations. Most of these patients were operated on under cocaine, but in some chloroform was necessary. The patient should be prepared for general anæesthesia and then operated on under local if possible. The removal of the anterior sphenoidal wall causes a good deal of pain. Probably the recumbent position is always advisable.

I wish now to make some remarks with reference to the examination of the eyes in these patients. This had special reference to the condition of the optic discs and was carried out in 14. In six the discs were reported normal; in four the condition was one of congestion of varying degree in the different patients and in either side of the same patient; in three there was definite optic neuritis on both sides. In two of these patients there was double sphenoidal sinusitis, while in one the disease was confined to the right side, but there was a small extradural abscess in connexion with the sinus disease. In this patient, though the neuritis was practically equal in degree on both sides at the time of the first examination, after the cure of the sinus disease the left disc improved more quickly than the right. In one case, that of a man with left sphenoidal and left posterior ethmoidal disease, there was atrophy of the left disc, while the right disc showed marked congestion. So that in these cases we have practically every gradation from the normal optic nerve to atrophy associated with disease of the posterior accessory nasal air spaces. Excepting the recent cases and in one patient who disappeared from observation, unfortunately one with optic neuritis, subsequent examinations were made at periods varying from five weeks to several months. The optic atrophy is progressive and the sight of that eye is getting worse. In all the others the cure of the sinus disease has been followed by cure of, or improvement in, the condition of the optic nerves. In view of the high percentage of cases in which these various changes in the fundus of the eye were found it cannot, I think, be controverted that they have the relation to the sphenoidal and ethmoidal disease of effect and cause. Whether the ethmoidal disease or the sphenoidal is the more baneful in this connexion it would be difficult to determine, and as a matter of fact the question is not of much practical importance, as the two diseases are so commonly associated. From the examination of a series of skulls I found that in the great majority the posterior ethmoidal cell did not extend back as far as the optic foramen. On the other hand, the intra-orbital part of the nerve which may extend to one inch has sometimes a very close relation to this cell, and, moreover, the cellular tissue which surrounds it and helps to protect it from injury rather makes it more vulnerable by permitting of the extension of the ethmoidal infection to the neighbourhood of the nerve. In rare instances from a developmental defect the nerve has been found actually traversing the sinus and the posterior ethmoidal cell, a fact which emphasises still more the necessity for great care in curetting the external wall. The relation of the posterior cell to the sinus is extremely variable, and in quite a fair proportion of cases there is a well-developed extension of the ethmoid along the external and upper boundary of the sphenoidal cavity. It is interesting to notice that in two of these patients, the one with optic atrophy and one of the patients with optic neuritis, this relationship was found.

How does the sinus disease secondarily affect the optic nerve? From what has been said it is evident that this may be by direct extension of the inflammatory process owing to a developmental or pathological defect in the bony wall and, as a matter of fact, the supero-external boundary is the part most likely to be affected by disease which may lead to destruction of the bone. In the case of the man with optic neuritis, nystagmus, and vertigo there was a fairly large gap in this part through which pus could be seen entering the sinus, an extra-dural abscess, in fact, in the region of the cavernous sinus and optic nerve. As a rule, however, I imagine the connexion between the changes in the nerve and the sinus disease is indirect. In many of the skulls I examined there were quite definite small openings leading from the sinus to the internal aspect of the base of the skull. These were specially seen in the external wall, but certain in the roof were fairly constant. The external layer of the etura mater is connected through these small foramina by thin fibrous bands with the under layer of muco-periosteum lining the sinus. In this way a cellulitis or an external pachymeningitis is set up by direct extensinn, and we get a sequence of events similar to that described by Gradenigo with reference to the involvement of the abducens nerve in the canal of Dorello secondary to middle-ear suppuration, the infection passing to the cellular tissue in the carotid canal and thence to the nerve. Similarly, the cellulitis or external pachymeningitis extends to the optic foramen, where the nerve gets a close enveloping sheath from the dura mater. In this connexion it is interesting to note that whereas it is rare (I only found it in 4 out of 230 skulls) to get one sinus so extensive as to come into direct relation with both the optic foramina, it is not so unusual (24 in 230) to get one sinus extending behind the other completely to the opposite side and coming into relation with both carotid 
tracts. This may be, and I have no doubt in some cases is, the explanation of changes in both optic discs with one-sided sinusitis, though another possible route of infection is through the roof to the optic commissure and tracts. In my experience, however, disease in the roof of the sinus is much less common than in the external wall. In rare instances the posterior ethmoidal cell of one side has been found extending backwards above both sphenoidal sinuses to the opposite side.

In conclusion, I would urge that in all cases of protracted post-nasal discharge, and especially in those cases where there is a condition of non-fœtid atrophy involving chiefly the posterior part of the turbinals, the possibility of chronic disease of the posterior group of accessory air spaces being the fons et orngo mali should be more clearly recognised. The diagnosis is not easy and in some cases can only be definitely made after remoral of the middle turbinate, a proceeding which one hesitates to carry out when the indications are not clear. Considering, however, the deleterious effects of a chronic post-nasal discharge, locally and on the general health, the bolder course will, I am convinced, more often be the wiser, and there should be no hesitation when an examination of the eyes reveals definite changes in the optic discs. Of late years attention has been drawn to the association of ocular and orbital inflammatory conditions with various nasal diseases so that it is hardly necessary to emphasise the necessity for an examination of the nose with special reference to posterior accessory sinus disease in cases showing vascular changes in the optic nerves.

Glasgow.

\section{MALIGNANT AND NON-MALIGNANT TUMOURS OF BILATERAL ORIGIN.1}

Bx W. ROGER WILLIAMS, F.R.C.S. ENG.

UNTIL, in a recent publication, ${ }^{2}$ I specially called attention to this subject by making a synthesis of many examples, which was the first of its kind, very little was heard among pathologists as to the bilateral origin of tumours. Yet no theory of neoplasia which fails to take into account this peculiarity can be regarded as really satisfactory. The wellestablished fact of the bilateral origin of tumours, although only an occasional occurrence, certainly indicates that tumour disease is something more than the manifestation of a solitary local aberration.

There can, I think, be no doubt that the activities of the local cells are largely conditioned by the totality of the forces which determine the integration of the whole organism, and the bilateral origin of tumours specially points to perturbation of these latter forces. In many anomalies per excessum, but specially in digital redundancy, when two or all four extremities may be simultaneously affected, we have an analogous condition; and underlying developmental disturbance of this general kind is probably the determining factor of most cases of bilateral tumour formation.

The chondromas furnish a good example of this. Thus, multiple cases are often met with in which the distribution of the tumours is bilateral, and, in exceptional instances, there may even be some approach to symmetry. In this type of disease it is by no means uncommon to find both hands affected with multiple tumours, and, in rarer cases, both hands and feet may be thus affected; while, quite exceptionally, most of the bones of the upper and lower limbs may be concurrently invaded with similar tumours, of which remark. able examples have been reported by Steudel, ${ }^{3}$ Kast, and others. The concomitancy of angiomatous growths with tumours of this kind is a significant item pointing in the same direction.

It accords with the foregoing that most bilateral tumours originate in early life, many of them being obviously the ontcome of gross developmental lesions of ante-natal origin. But, besides these ante-natal causative factors, it appears that certain acquired morbid conditions, such as chronic

1 A paper read before the Bristol Medico-Chirurgical Society on Jan. 12 th, 1910 The Natural History of Cancer, 1908, p. 298

3 Bruns' Beiträge zur klinischen Chirurgie, Band viii., S. 503. eczema, seborrhœa, and other hyperplastic lesions, some. times determine bilateral neoplasia.

Among the numerous instances of the multiple outbreak of malignant growths lately reported, some of the most striking are those in which the malady arises concurrently in both of paired organs, or in both of other bilaterally symmetrical parts of the body. These bilateral tumours are often described as symmetrical, but it is seldom that they correspond so exactly as to justify the use of this term.

It is of interest to note that non-malignant tumours of bilateral origin are even less exceptional than their malignant congeners ; thus, papillomas, angiomas, moles, fibromas, odontomas, myomas, adenomas, chondromas, osteomas, dermoid, sebaceous and other cysts-all present bilateral manifestations, and not so very rarely. An instance of generalised nævi, in which the distribution of the lesions seemed to indicate metameric symmetry, has been described by Hallopeau and Weil," and several cases of "fissural" angioma are on record. Hutton and others have reported examples of bilaterally generalised angioma, and hereditary instances of this kind have been studied by Parkes Weber, Osler, and others.

Among the fibromas, striking examples of bilaterality are met with in the disease called "multiple fibroma of nerves," in which over two thousand tumours have been counted in a single patient; and, in exceptional cases, the grouping of the tumours may be symmetrical. Although this disease is usually met with in adults, many early-life cases have been recorded, in some of which the tumours were of ante-natal origin. Probably the germs of this disease always are congenital, the tumours arising in defectively developed areas of the endoneurium. In this connexion it is worth noting that in a considerable proportion of cases, one or more of the numerous tumours eventually take on sarcomatous change. Moreover, various other developmental defects may be met with, in those who are subject to this disease, which affects horses, oxen, and other cattle, as well as mankind.

In the condition commonly called "diffuse lipoma" we meet with multiple, bilateral, and symmetrical tumours, but these formations can hardly be regarded as true tumoursthey are rather the outcome of a tropho-neurotic process. The supra-clavicular fatty masses, ${ }^{6}$ which are usually bilateral, belong to the same category; and here also "lipoma arborescens" may be included. To indicate this distinction it is best to speak of these overgrowths as examples of diffuse "pseudo-lipomatosis."

Among the true lipomas bilateral forms are met with which are occasionally symmetrical, but such occurrences are decidedly rare. Spencer Wells ${ }^{7}$ successfully removed a fatty tumour, weighing $16^{\frac{1}{2}}$ pounds, from one perirenal region; and a similar tumour, weighing $14 \frac{1}{2}$ pounds, from the corresponding region on the opposite side. Doran ${ }^{8}$ found bilateral retroperitoneal lipoma of the posterior abdominal wall; Senn ${ }^{9}$ and others lipoma of each axilla. Examples of bilateral lipoma of the tendon sheaths of the feet are also on record. Of lipomas in hernial positions, J. Hutchinson, jun., and others have reported bilateral examples.

Among the many other instances of bilateral lipoma reference may be made to some striking cases by A. Prichard, ${ }^{10}$ in which the limbs-chiefly the upper ones-were the seat of symmetrical tumours. Cruveilhier had previously described a similar case.

The most remarkable example of this kind known to me was reported some years ago by Broca. ${ }^{11}$ His patient was a man, aged 70 years, who when he was only 25 years old first noticed a small fatty tumour at the upper and outer part of his right hip. In the course of six years it gradually attained a large size. It was then extirpated and found to be an ordinary fatty tumour. At this time the patient had no other tumour. The wound healed, and there was no sign of any return of the disease. However, at the end of five months an eruption of hundreds of small fatty tumours gradually took place all over his body. Most of these remained quite small. Fresh tumours continued to form in this way for

\section{Aunales de Dermatologie, tome viii., p. 483.
5 THe LanceT, July 20th, 1907 , p. 160.}

6 For further information on this subject reference may be made to the writer's publication on Diffuse Pseudo-lipoma (Transactions of the Pathological Snciety, vol. xli., p. 289).

Brit. Med. Jour., 1889, vol, i., p. 757

Transactions of the Obstetrical Society, 1902, p. 265

9 Pathology and Surgery of Tumours, 1900 , p. 406

10 Brit. Med. Jour., 1881, vol. i., p. 271.

11 Traité des Tumeurs, tome i., p. 304. 